\title{
PRESENTACIÓN
}

\section{Legislación basada en evidencia científica. El caso de México}

A ctualmente, un núcleo informado de la población demanda una respuesta eficiente de las instituciones que conforman un Estado para implementar políticas públicas de salud basadas en evidencia científica, fundamentada en dos hechos principales: garantizar un impacto en la salud poblacional y promover la participación activa de la sociedad civil en el proceso de toma de decisiones. A este respecto, a pesar del escaso número de leyes que se promueven periódicamente para avanzar en salud en el ámbito regional, las evidencias que proporciona la investigación han sido escasamente utilizadas en los procesos de construcción de políticas de protección pública. Existen evidencias en la literatura científica que sugieren que los políticos tienen limitado tiempo e incentivos para conocer detalles de la información científica generada y por lo general necesitan del apoyo de expertos de áreas sustantivas. La reputación y credibilidad de los grupos de investigación, así como su relevancia local, son importantes para que los responsables de generar las nuevas políticas públicas los tomen en cuenta. Por ello, los resultados de investigación deberían tener la más alta influencia en los debates públicos para la creación de nuevas leyes que impacten en salud pública. Sin embargo, el grado de aceptación y uso de los resultados de investigación dependen del contexto sociopolítico en que se desarrolle la discusión, el impacto que los propios hallazgos de investigación generen, así como la motivación política para generar los cambios en los paradigmas de prevención y promoción de la salud.

La traducción de la investigación científica en impacto comunitario depende en gran medida de la claridad que tengan los diversos actores para generar, difundir, transmitir, traducir, absorber, utilizar y aplicar el conocimiento científico. Este ejercicio de comunicación requiere de una promoción sistemática de interacción entre grupos de investigación y tomadores de decisiones, en donde los profesionales de comunicación de investigación en salud pública y abogacía tienen una participación sustantiva.

Evidencia obtenida de la experiencia en México para el desarrollo de políticas públicas en control del tabaquismo, indican que el conocimiento preciso de los legisladores en relación con los efectos biológicos, económicos, culturales y sociales de la exposición activa y pasiva al humo del cigarro fue el principal fundamento para el desarrollo de una legislación de vanguardia para el control del tabaquismo. Existen evidencias en México de que no sólo el conocimiento, sino el valor emocional de la información de los efectos del tabaquismo en el ámbito poblacional lograron tener un valor intrínseco para asumir una posición responsable en la toma de decisiones.

Dada la importancia crítica de políticas basadas en evidencia científica para avanzar en la prevención y control del tabaquismo, así como otros aspectos de salud poblacional, los considerables obstáculos para efectivamente traducir los resultados de investigación en políticas y entender mejor cómo superar las barreras actuales y futuras, en este número monográfico de la revista Salud Pública de México, titulado "Abogacía en salud pública para el control del tabaco en México", diferentes autores nacionales e internacionales exploran la intersección entre investigación y desarrollo de políticas, enfatizando no sólo el estudio de caso del control del tabaquismo en México, sino también los retos que 
se enfrentarán en el futuro para implementar la nueva legislación que promueve ambientes libres de humo de tabaco, considerando la experiencia internacional.

Este ejercicio es inédito en nuestro contexto, debido a que los profesionales de la salud y los tomadores de decisiones tienen un muy limitado entrenamiento para buscar eficientemente información relevante y objetiva para su traducción e implementación en políticas públicas. La información de alta calidad y objetividad no está fácilmente disponible. Sin embargo, para el caso de la caracterización del estudio del tabaquismo en México, existieron diversos antecedentes que fueron útiles para fundamentar la nueva legislación: los números monográficos de las revistas de salud pública publicados durante los últimos años, los resultados de las encuestas nacionales de Salud y Adicciones, así como las de Tabaquismo y Juventud, y el informe del secretario de Salud para el control del tabaquismo en México, primer antecedente en su género originado en un país en vías de desarrollo y coordinado por el Instituto Nacional de Salud Pública. Los anteriores, son ejemplos de la interacción que grupos de investigación nacionales e internacionales ofrecieron como fundamento a la toma de decisiones de los legisladores.

Los investigadores teníamos la percepción de que los tomadores de decisiones no estaban suficientemente preparados para tomar una decisión explícita basada en evidencia científica, porque la mayoría de las ocasiones estas decisiones fueron tomadas por un proceso de asignación histórica o por un sesgo ideológico-político. Con la generación de una ley contra el tabaquismo, este estigma hacia los legisladores ha sido modificado. El establecer prioridades de políticas en salud puede estar sujeto a criterios múltiples y la toma de decisiones se vuelve muy compleja. A este respecto, las intervenciones deberían ser elegidas para maximizar la salud de la población general, reducir inequidades en salud de los grupos marginados o vulnerables, y/o dar respuesta a situaciones de emergencia. En este entorno, tradicionalmente los tomadores de decisiones tendían a utilizar aproximaciones intuitivas para tratar de simplificar la complejidad de las mismas, y durante este proceso muchos de ellos ignoraban evidencias científicas que constituían un elemento central en la solución de los problemas. En resumen, en nuestro medio durante mucho tiempo las nuevas propuestas legislativas en salud eran seleccionadas con una fuerte motivación política. Por esta razón, actualmente el foco primario de asignación de prioridades debería ser la toma de decisiones basada en evidencia científica, donde el elemento clave para mejorar las políticas de salud debe ser la obligación de rendir cuentas a todos los miembros de la sociedad.

Diversos abordajes conceptuales han sido utilizados para organizar la base de la evidencia para asegurar y tener el más alto impacto en salud, y generar un juicio sobre el impacto en una política de salud, programa social o proyecto comunitario, además de analizar cómo se distribuyen dichos efectos. Han sido reconocidas diversas maneras en que la evidencia sobre la salud y sus determinantes pueden ser relacionados con las políticas de salud y muchos ejemplos se han ofrecido en la literatura científica. La más completa es el análisis de los efectos en salud en el contexto de una comparación de opciones. Otra evidencia que se ha cuantificado es la de correlacionar cambios en los niveles de exposición o de otros factores de riesgo con el estatus de salud. Invariablemente, en estas opciones no sólo será necesario identificar la evidencia científica sino que la evaluación continua será una forma de garantizar la rendición de cuentas. En este contexto, la principal brecha que hay que superar es la necesidad de integrar eficientemente las opciones de políticas de salud con los cambios en los determinantes de salud. Finalmente, en México se debe acreditar en forma rutinaria la toma de decisiones basada en evidencia científica, y para tal efecto los legisladores, así como los profesionales de la salud deben entrenarse para este fin. Sólo desde esta perspectiva estarán respondiendo en forma organizada a la gran encomienda y responsabilidad que les ha sido conferida.

Dr. Eduardo Lazcano-Ponce* Dr. Éctor Jaime Ramírez-Barba ${ }^{\ddagger}$

\footnotetext{
* Instituto Nacional de Salud Pública, México.

‡ Universidad de Guanajuato. Hospital Ángeles León. Presidente de la Comisión de Salud de la Cámara de Diputados, México.
} 